\title{
Eye Tracking in Human-Computer Interaction and Usability Research
}

\author{
Tommy Strandvall \\ Tobii Technology, Karlsrovagen 2D, 18253 Danderyd, Sweden \\ tommy.strandvall@tobii.com
}

\begin{abstract}
The objective of the tutorial is to give an overview on how eye tracking is currently used and how it can be used as a method in human computer interaction research and especially in usability research. An eye tracking system records how the eyes move while a subject is completing a task for example on a web site. By analyzing these eye movements we are able to gain an objective insight into the behavior of that person.
\end{abstract}

Keywords: eye tracking, eye movements, usability, human-computer interaction, method, methodology.

\section{Tutorial Overview}

The objective of the tutorial is to give an overview on how eye tracking is used and can be used as a method in human computer interaction research and especially in usability research. By analyzing eye movements collected by an eye tracking system we are able to gain an objective insight into the behavior of a person and present the results as powerful visualizations or eye movement metrics and statistics.

During the tutorial we will present a range of eye tracking metrics and visualizations and explain how these can be interpreted in the context of interface design and usability evaluation. Examples of such eye movement metrics are; Number of Fixations, Fixation Duration, and Time to First Fixation. Common ways of visualizing eye movements in a usability or HCI setting are; Heat Maps, Gaze Plots or Scan Paths, and Gaze Replay Videos. Using these eye tracking metrics and visualization together with existing research methods can reveal insights previously unavailable to researchers and practitioners. Additionally it allows us to develop new approaches and methodologies. Some new methodologies where eye tracking plays an important role have already been developed like in the case of the Post-Experience Eye-Tracked Protocol (PEEP) [1].

The tutorial will moreover include hands-on eye tracking tests and analysis exercises. This tutorial is appropriate for researchers, practitioners and students. Participants should be familiar with behavioral research methodology but no prior knowledge of eye movements or eye tracking is required. 


\section{Introduction to Eye Movements}

An eye tracking system records how the eyes move when a subject is sitting in front of a computer screen. The human eyes are constantly moving until they stop and focus on a point. There are over ten different types of eye movements, of which the most important ones are saccades and fixations. When the eyes focus on a point it is called a fixation and the movements between these fixations are called saccades. When the eye fixates, the stops vary from about 100 to 600 milliseconds and during this stop the brain starts to process the visual information received from the eyes. The length of a fixation is usually an indication of information processing or cognitive activities as this is when the brain interprets the visual information from the eyes. The human eye has a visual field of about $200^{\circ}$ but the highest number of light sensitive cells on the retina are located in the part called fovea, which is the only point in our eyes where we are able to see a sharp and colorful image of the world around us. This area is fairly small and covers only about $1-2$ degrees of our vision (which is about the size of a thumbnail at an arm length's distance). It is only from these cells our brain can receive detailed visual information. An eye tracker records these movements and the location of the foveal vision when the eyes fixate. [2]

It is possible to move our covert attention (the attention of our mind) around the entire visual field when our eyes are at rest. Thus it is possible to move our attention around without eye movements. However, our foveal vision is usually a valid measure for determining the target of our covert attention as our brain can process very little information from complex stimuli from the area outside the fovea (such as objects on a website or software interface) [3]. In such cases it is more efficient for our brain to focus the attention on the fovea rather than on our peripheral vision, as the brain needs to process blurry visual information requiring more effort to interpret than the visual information from the fovea.

This is why it is possible to tell something about human behavior by just following eye movements, especially the fixations, as we know that we can only see something clearly when we fixate on, or near, an object.

\section{References}

1. Ball, L., Eger, N., Stevens, R., Dodd, J.: Applying the post-experience eye-tracked protocol (PEEP) method in usability testing. In: Interfaces, Informs, Cincinnati, vol. 67, pp. 15-19 (2006)

2. Rayner, K.: Eye Movements in Reading and Information Processing: 20 Years of Research. Psychological Bulletin 124, 372-422 (1998)

3. Pieters, R., Wedel, M.: Informativeness of eye movements for visual marketing: six cornerstones. In: Wedel, M., Pieters, R. (eds.) Visual Marketing: From Attention to Action. Lawrence Earlbaum, New York (2008) 